\title{
La Investigación Socio Territorial: Una Propuesta para Comprender los Procesos de Inclusión de los Migrantes en las Escuelas
}

\author{
Socio-territorial Research: A Proposal to Understand the \\ Processes of Inclusion of Migrants in Schools
}

\author{
Natalia Salas * \\ Felipe Kong \\ Renato Gazmuri
}

Universidad Diego Portales

\begin{abstract}
En el presente artículo, se pone el foco en la migración hacia Chile, especialmente de los niños, niñas y adolescentes que ingresan al sistema educacional chileno y el uso de la investigación socio territorial como una forma de analizar la construcción de identidades, que son dinámicas e influyen en la inclusión educativa de los mismos. Una de las metas del sistema educacional chileno dice relación con brindar un ambiente educativo de calidad, donde equitativamente todos los estudiantes puedan alcanzar su potencial académico. En ese contexto, uno de los tópicos recurrentes en el debate sobre migración y escuela es la tensión existente entre la consolidación de prejuicios y estereotipos o la capacidad de la institución escolar para modificarlos. En este artículo se identifican los aportes del enfoque socio territorial para la comprensión de la migración, así como para orientar el diseño de políticas públicas y de las estrategias de las escuelas para enfrentar los desafíos de la migración.
\end{abstract}

Descriptores: Educación de los inmigrantes, Identidad cultural, Educación integradora, Segregación racial, Sociología urbana.

In this article, the focus is on migration towards Chile, especially the children and adolescents entering the Chilean educational system and the use of socio-territorial research as a way of analyzing the construction of identities, which are dynamic and thus influence the educational inclusion of them. One of the goals of the Chilean educational system is related to providing a quality educational environment, where all students can equitably reach their academic potential. In this context, one of the recurring topics in the debate on immigration and school is the tension between the consolidation of prejudices and stereotypes or the capacity of the school to modify them. This paper highlights the importance of socio territorial studies to understand immigration, and how these progressively influence spatial groups (school community and migrants) and representations / prejudices associated to them.

Keywords: Migrant education, Cultural identity, Inclusive education, Racial segregation, Urban sociology.

Esta publicación utiliza información y se enmarca dentro de un proyecto financiado por el Ministerio de Educación de Chile, el cual contó con el financiamiento del Fondo de Investigación y Desarrollo en Educación (FONIDE $N^{\circ}$ F91 1424, año 2014).

*Contacto: natalia.salas@mail.udp.cl

ISSN: 0718-7378

www.rinace.net/rlei/

$\begin{array}{ll}\text { Recibido: } & 14 / 12 / 2016 \\ 1^{a} \text { Evaluación: } & \text { 20/01/2017 } \\ \text { Aceptado: } & 22 / 03 / 2017\end{array}$




\section{Enmarcando el problema: El fenómeno migratorio en Chile}

La migración se define y/o conceptualiza como el desplazamiento de personas o grupos de personas. La Organización Internacional para las Migraciones (OIM, 2006) se refiere al mismo como el "movimiento de población hacia el territorio de otro Estado, o dentro del mismo, que abarca todo movimiento de personas sea cual fuere su tamaño, su composición o sus causas; incluye migración de refugiados, personas desplazadas, personas desarraigadas, migrantes económicos" (p. 38). Por lo general, las migraciones están asociadas a desplazamientos motivados por la satisfacción de necesidades que favorezcan las condiciones de vida (OIM, 2007), situación que se observa en Chile.

En el presente artículo, se pone el foco en la migración hacia Chile, es decir, en el "proceso por el cual personas no nacionales ingresan a un país con el fin de establecerse en él” (OIM, 2006, p. 32). Este proceso está caracterizado por ser voluntario, por la búsqueda de una mejor calidad de vida; y en donde la decisión de migrar es tomada libremente, sin la intervención de factores externos que lo fuercen a hacerlo (como lo es el caso de la trata humana o la migración por conflictos armados).

Por tanto, la migración es entendida como un proceso de construcción social, que al ser dinámico, moviliza y que se relaciona estrechamente con la realidad subjetiva de la persona que migra (Schwartz et al., 2013; Veredas, 1999). En este sentido, el migrante construye una identidad en torno a su proceso de migrar, no solo desde las condiciones socio-económicas previas, sino también, desde una mirada de nueva territorialidad.

De este modo, en el ámbito de los estudios migratorios no solo se considera relevante la construcción identitaria del sujeto como tal, sino también de los miembros de su familia, particularmente aquellos que nacen en el nuevo contexto cultural-territorial, como los hijos, quienes de alguna manera deben decidir "de dónde son" (Schwartz et al., 2013; Berry y Vedder, 2016). Al respecto, Portes (1997) releva las dificultades que enfrentan los hijos al crecer en una familia de migrantes, debido a que éstos buscan equilibrar la orientación de sus padres extranjeros con las demandas de "asimilación" de la sociedad y el territorio que lo recibe.

Estudios realizados a nivel local/nacional respecto a migrantes latinoamericanos (especialmente la comunidad peruana), dan indicio de que éstos son visualizados a partir estereotipos (asociados a raza, pobreza, delincuencia, entre otros) que operan como fuente de discriminación (Arias, Moreno y Nuñez, 2010; Tijoux, 2013). De este modo, ser hijo(a) de migrante se considera diferente a lo local, lo cual conduce a estigmas al ser identificado como un "otro/migrante" diferente a "nosotros/nacionales" aun cuando se trate de niños(as) que nunca han migrado espacialmente o que no son extranjeros en términos legales (Goffman, 2001; Olmos, 2012; Tijoux, 2013).

Las migraciones hoy se insertan en procesos más amplios, de carácter planetario, de movilidad y globalización, que no solo implican una traslación física de quien la experimenta, sino que también, incorpora complejos factores económicos, políticos, culturales y sociales que la promueven (Castells, 2001; Mähönen y Jasinskaja-Lahti, 2016). 
En este escenario, y a partir de la década de los 90 se ha generado un incremento en el flujo de migración intra-regional en América Latina, siendo Chile uno de los polos más atractivos para los migrantes sudamericanos (González, 2009; Sirlopú et al., 2015) y recientemente (CASEN, 2013) se diversifica su procedencia a países del Caribe (por ejemplo, Haití y República Dominicana). En efecto, entre 1992 y 2002 (INE, 2003) la migración de latinoamericanos a Chile creció en un $75 \%$, porcentaje más alto durante los últimos 50 años (Ministerio del Interior, 2004). Actualmente este grupo de migrantes representa aproximadamente el 2,3\% del total de población (INE, 2015). A pesar de que dicho porcentaje parezca bajo (en comparación con migrantes de países desarrollados, donde alcanza un 11,3\%) demuestra un crecimiento de un punto porcentual desde el Censo 2002 (OBIMID, 2016). Este movimiento migratorio, está motivado principalmente por la estabilidad económica y política del país (OECD, 2012; OBIMID, 2016), siendo la Región Metropolitana quien concentra mayor porcentaje, con un $61,5 \%$ de la población migrante nacional, según estadísticas del INE (2015); en término de visas temporales (migración reciente) el OBIMID (2016) destaca que para el año 2015 hubo un crecimiento porcentual del $21 \%$ en relación con el 2014.

Tal crecimiento de población migrante presenta concentración en ciertos establecimientos educativos, especialmente en la red pública (51\%, de acuerdo a la CASEN en 2006) (Pavez, 2012). Sin ninguna duda, el contexto educativo no queda ajeno a la realidad de la migración, una de las áreas de la política de inclusión promovida desde el MINEDUC incluye específicamente el trabajo con la población migrante, lo que se refleja en instancias conjuntas entre el Ministerio de Educación y el Departamento de Extranjería, que tienen por objeto facilitar y promover la incorporación de todos los hijos de migrantes que residen en Chile a los diversos establecimientos de educación general básica y media, independiente de la condición migratoria de sus padres (MINEDUC, 2005). Según estos datos, se observa que para el año 2005 habían 0.3\% estudiantes migrantes y para el año 2012 ya había un aumento hacia un 1\%; estando presentes en los diferentes niveles (35\% del total de establecimientos del país); así como en los diferentes tipos de dependencia (para el 2016 60\% de los/as estudiantes migrantes se encuentran en el sector municipal, $34 \%$ en el sector particular subvencionado y $6 \%$ en establecimientos particulares pagados).

Respecto del marco legislativo nacional, y a pesar de que la Ley de Extranjería (creada en 1975 y vigente a la fecha) limita y posee una mirada sumamente restrictiva de la migración, se han desarrollado una serie de decretos y leyes que aseguran el acceso y derecho a educación a todos(as) los/las estudiantes de edad a la educación básica y media., incluso para aquellos que se encuentran en situación irregular (tal es el caso de la circular 1179, el decreto 597 y el ordinario $\mathrm{N}^{\circ}$ 07/1008). A su vez, desde el año 2005, y por medio de la ratificación de la Convención Internacional sobre la protección de derechos de todos los trabajadores migratorios y sus familiares, se plantea la necesidad de incorporar al sistema educacional a los niños, niñas y adolescentes migrantes, independientes de su condición migratoria o la de sus padres. Sin embargo, esto implica que las políticas educativas en Chile se limitan a asegurar el acceso de los niños/as y adolescentes inmigrantes a las escuelas, pero no necesariamente de asegurar un proceso de inclusión. Si bien es posible identificar algunas experiencias de carácter local y otras que se han realizado por las propias escuelas donde se hace una buena inclusión de la diversidad cultural, estas se establecen de manera general, sin ser sistematizadas. Recién en el año 2015 (Instructivo 005) y en el 2016 (actualización del Ordinario No.07/1008), 
se plantean nuevos lineamientos para gestionar la política nacional migratoria, incluyendo aspectos sobre la inclusión del escolar migrante (como el programa Escuela somos todos, Visa Migrante o más recientemente el identificador único para escolares migrantes).

\section{La investigación sobre migración y su efecto en el sistema escolar}

Los estudios sobre la situación de los migrantes se han focalizado principalmente en temáticas tales como el género, las condiciones de trabajo y la niñez, y muestran evidencia de alta discriminación por parte de la sociedad chilena (Pavez 2012; Sirlopú et al., 2015; Stefoni et al., 2010; Tijoux, 2013, 2014); así como, la experiencia de los/as migrantes en el sistema educativo chileno, especialmente desde la mirada de la educación intercultural y las estrategias de participación (Berrios-Valenzuela y Palou-Julian 2014; Donoso, Mardones y Contreras, 2009). Al respecto, Alegre, Benito y González (2006), desde una perspectiva más cercana al mundo escolar, sostienen la necesidad de analizar las experiencias sociales de los sujetos inmigrantes a partir de los posicionamientos culturales, identificando múltiples factores y dinámicas en juego que explican dichos procesos de incorporación. Concluyen, a continuación, que el espacio escolar es un terreno privilegiado para observar los mapas culturales y relacionales que se producen entre migrantes.

Pese a esto, cabe indicar que existe poca evidencia sobre las características, perfiles y forma en la cual viven sus procesos de escolarizaciones el conjunto de los niños, niñas y adolescentes migrantes. Entender los procesos migratorios donde niños, niñas y adolescentes se ven involucrados, resulta ser un tema complejo de abordar, tanto en términos de adaptación como de inclusión (Stefoni, 2011).

Los estudios realizados en Chile, respecto de la migración y la escuela, presentan dos miradas de indagación. La primera mirada pone foco en las políticas públicas entorno al fenómeno migrante, desde el enfoque de los derechos humanos. En estos estudios se observa que persisten dificultades para la integración del alumnado interculturalmigrante, principalmente por la falta de recursos y formación en la educación chilena; así como la ausencia de lineamientos que clarifiquen cómo aproximarse al fenómeno, consecuencia de una ley migratoria deficitaria (Berrios-Valenzuela y Palou-Julian, 2014; Donoso, Mardones y Contreras, 2009; OIM, 2007; Stefoni et al., 2010; Tijoux, 2013).

La segunda mirada se refiere a la perspectiva que poseen los mismos actores: escolares migrantes, sus profesores y sus familias. Estos estudios abordan, mayoritariamente, la percepción desde los conceptos de identidad y discriminación especialmente cuando la migración se presenta en la infancia (Mardones, 2010; Pavez, 2012). Los resultados de dichas investigaciones muestran procesos de cambio que las escuelas realizan para crear practicas inclusivas adaptando sus propios proyectos de integración, como por ejemplo en la realización de actividades extra-curriculares de tipo recreativas, o folklorizantes (Moreno y Oyarzún, 2013; Navas y Sánchez, 2010; Poblete Acuña y Zegers Quiroga 2014). 


\section{Prejuicio y educación}

En concordancia con los estudios revisados, se han encontrado patrones de discriminación y prejuicio hacia aquellos estudiantes migrantes (Berrios-Valenzuela y Palou-Julian, 2014; Stefoni, Acosta, Gaymer y Casas-Cordero, 2008; Sirlopú et al., 2015). Entenderemos al prejuicio como una actitud (normalmente negativa) hacia los miembros de algún grupo, basada solo en la pertenencia a un grupo social (Baron y Byrne, 2005; Stangor, 2009). Esta actitud incluye tanto evaluaciones negativas acerca del grupo social, como también sentimientos y emociones negativas hacia ellos (Bodenhausen, Kramer y Susser, 1994). A su vez, los prejuicios también pueden incluir creencias y expectativas sobre los miembros de estos grupos, concretamente estereotipos, que sugieren que todos los miembros de estos grupos poseen ciertas características y actúan de una forma determinada (McGarty, Lala y Douglas, 2011).

Un extenso cuerpo de investigaciones ha mostrado que los prejuicios pueden tener un impacto negativo en los sujetos a quienes se dirigen (Degner y Wentura, 2010; Dovidio, 2001; Lowery, Hardin y Sinclair, 2001; Tropp, 2003) a partir de sus componentes (actitudes y conductas); especialmente cuando emergen dentro del aula (Aronson, Quinn y Spencer, 1998; Rodríguez y Retortillo, 2006). Estas expectativas y prejuicios, se van consolidando con la experiencia y poca reflexión pedagógica, provocando formas inadecuadas de actuar frente a la diferencia. Respecto de la actuación frente a temas de inmigración, Navas, Holgado y Sánchez (2009), sugieren que la valoración de la educación intercultural que posea el educador, influye en el trato e interacción del mismo para con sus estudiantes migrantes. A su vez, ésta valoración puede predecir la atribución de estereotipos académicos.

Por otra parte, autores como Hitti, Lynn Mulvey y Killen (2011), a partir de las opiniones y percepción de escolares sobre exclusión en diferentes contextos sociales, identifican a la etnia y la cultura como variables relevantes de considerar como factores que contribuyen a legitimar o rechazar a los miembros de los grupos externos. Dado lo anterior, este artículo busca enlazar la dimensión cultural desde la mirada de los procesos de cognición social y territorial asociados. Esto resulta particularmente preocupante, dado que muestra construcción de discriminación desde etapas escolares; así como identifica que el hecho de poseer una alta valoración por las características del grupo de origen, en contraposición con grupos culturalmente externos, como es el caso de los escolares migrantes conlleva a formas implícitas de prejuicio (Salas et al., 2016); así como, a que existan atribuciones de estereotipos negativos hacia los pares de distinta nacionalidad, ya sea por ser originarios de un país diferente o porque sus progenitores sean migrantes (Enesco et al., 2011; Hitti, Lynn Mulvey y Killen, 2011; Solbes et al., 2011; Tijoux, 2014).

Uno de los tópicos recurrentes en el debate sobre inmigración y escuela es la tensión existente entre la consolidación de prejuicios y estereotipos o la capacidad de la institución escolar para modificarlos. Cárdenas y colaboradores (2011) detectó en una muestra de estudiantes de enseñanza media que los prejuicios abiertamente hostiles habían derivado en otros más sutiles o encubiertos, pero no desaparecidos, corroborando la hipótesis de Pettigrew y Meertens (1995) a este respecto, de que existe dificultad en reconocerse a sí mismos como racistas o xenófobos. Para el caso español, Thayer (2008) detectó que la estigmatización al interior de los espacios escolares es menor si la cantidad de inmigrantes es mayor, esto fue corroborado en Chile por Salas y sus 
colaboradores (2016). El estudio registró los prejuicios implícitos y explícitos de escolares de cuarto año básico, respecto de sus pares migrantes. Los resultados muestran que existen prejuicios ya desde la edad escolar, y que éstos aumentan o disminuyen a partir de las posibilidades de contrastar las representaciones que se poseen respecto del migrante con aquellas vivenciadas directamente. Específicamente, el estudio concluyó que en aquellas escuelas donde el porcentaje de escolares migrantes era alto (superior al $40 \%$ ) el prejuicio disminuía; mientras que en aquellas escuelas donde existían pocas probabilidades de interactuar con un par migrante, y donde había un bajo porcentaje de escolares migrante (menos del 10\%), dichos prejuicios aumentaban. Este prejuicio sutil también se detectó en estudiantes de pedagogía en Chile, con menor intensidad en mujeres y personas con interacciones previas con inmigrantes (Navas y Sánchez, 2010; Tijoux, 2013).

En suma, el proceso de inclusión de la población migrante se ha enfrentado a desafíos y dificultades en distintas partes del mundo. Las políticas públicas y sociales, así como las estrategias escolares diferenciales, han generado distintos procesos de incorporación de estos colectivos a las comunidades de recepción. Los modos en que estos procesos se han desarrollado en Chile, no han permitido que exista aún evidencia sólida que oriente adecuadamente las políticas públicas.

A continuación se revisa la relación entre las propuestas de interculturalidad, lo que el modelo de inclusión educativa propone y lo que se observa en el contexto educacional, especialmente desde lo curricular, acerca de la condición intercultural migrante.

\section{Interculturalidad, inclusión y educación}

Dados los antecedentes de prácticas de exclusión revisados anteriormente, emerge en el año 2005, una primera aproximación al trabajo con estudiantes de diversidad cultural, especialmente de niños/as migrantes

.... visibilizando y valorando las diferencias individuales, contemplando respuestas educativas de calidad para todos los alumnos, facilitando la atención de la diversidad social y cultural, con el fin de reducir o minimizar las dificultades de aprendizaje que tienen su origen en una enseñanza que no considera las diferencias individuales. (MINEDUC, 2005, p. 36)

Surge entonces un ímpetu hacia un enfoque de la Educación Inclusiva, la que pone el foco

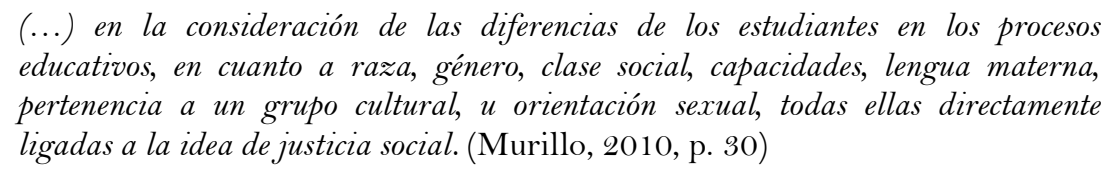

Tal como plantean Booth y Ainscow (2012), la transformación de la institución escolar parte por crear culturas inclusivas, elaborar políticas inclusivas y desarrollar prácticas inclusivas, es decir, asumir un desafío que permita levantar barreras para el aprendizaje, acceso y participación que se generan en los sistemas educativos. Esto es respaldado por UNESCO (1994), para quienes las escuelas con orientación inclusiva se convierten en el medio y espacio por naturaleza para modificar actitudes discriminatorias, gestar comunidades de acogida, construir una sociedad más inclusiva y lograr efectivamente una educación para todos. 
La meta de la educación inclusiva, es por lo tanto eliminar la exclusión social que se deriva de actitudes y respuestas a la diversidad (Blanco, 2006). Tal como lo plantea Theoharis (2007), aquí está implícito el derecho a la educación ligado a los valores de justicia y democracia, interpelando a la escuela para ser una institución más justa y equitativa. En este sentido, es importante que los docentes tienen "en sus manos [...] la clave del aprendizaje intercultural" (Zafra, 2011, p. 1).

Por tanto, el aumento de la inclusión implica reducir la exclusión, a partir de un proceso continuo y sistemático donde el aprendizaje y la participación de todos/as, se transforma en un horizonte a alcanzar (Booth y Ainscow, 2012; Echeita et al., 2009). Un contexto inclusivo puede ser descrito como aquel que está en movimiento y busca abordar la tendencia a la exclusión que tiene. En el sistema educativo, hay muchas presiones que favorecen la exclusión, son persistentes y pueden adoptar nuevas formas y expresiones culturalmente situadas (Ainscow, Booth y Dyson, 2006). La inclusión emerge tan pronto el establecimiento educativo reconoce los factores de exclusión que posee y establece un plan de mejora de la participación (Donoso, Mardones y Contreras, 2009). La implementación de políticas inclusivas parte de ese reconocimiento, a través de herramientas analíticas y descriptivas, que permitan caracterizar los establecimientos, respecto de las características culturales que poseen, y dentro de estas aquellas referidas a lo socio territorial y a las adaptaciones curriculares ante el desafío interculturalmigrante

Como se ha planteado, una de las metas del sistema educacional chileno dice relación con brindar un ambiente educativo de calidad, donde equitativamente todos los estudiantes puedan alcanzar su potencial académico (Guerrero, Provoste y Valdés, 2006). Autores como del Río y Balladares (2010) proponen que un posible origen de los estereotipos antes mencionadas reside en las interacciones que ocurren en los contextos educativos y en las actitudes de los individuos involucrados en el proceso de enseñanza/aprendizaje.

En un estudio realizado por Steinmayr y Spinath (2008), se encontró que si los profesores sostienen la creencia de que los logros académicos de sus estudiantes están determinados por factores más allá de su control (por ejemplo la cultura de la cual proviene), mostrarán una baja motivación para utilizar estrategias que mejoren el logro de estos estudiantes; así como sostendrán percepciones prejuiciosas de sus estudiantes (Ortiz, 2008). Esto fue corroborado por autores como León, Mira y Gómez (2007), a través de una evaluación de las opiniones sobre la inmigración y la multiculturalidad en la escuela realizada a alumnos del magisterio en España.

Para hacer frente a dichos prejuicios, algunos autores señalan la mirada intercultural, como una que apoyaría la eliminación de dichas manifestaciones (Shmelkes, 2004; Veredas, 1999). La interculturalidad hace referencia a un concepto dinámico que aborda analíticamente las relaciones, negociaciones e intercambios entre grupos culturales que conviven en un mismo espacio (Aguado y Ariosa, 1991; UNESCO, 2005; Walsh, 2005). Este concepto ha sido definido como "la presencia e interacción equitativa de diversas culturas y la posibilidad de generar expresiones culturales compartidas, adquiridas por medio del diálogo y de una actitud de respeto mutuo" (UNESCO, 2005, p. 8). Es relevante considerar que tanto los prejuicios como las actitudes tienen impacto en el tipo y calidad de la interacción cultural y en la forma que la inclusión educativa proponga (Avramidis y Norwich, 2002). La interculturalidad implica la creación y asunción de actitudes entre todos los miembros de la comunidad educativa que favorezcan la convivencia entre 
personas de diferentes etnias, culturas y razas. La escuela se convierte en un espacio privilegiado para potenciar la convivencia entre los pueblos, e inculcar el valor positivo de la diversidad, siendo la respuesta a la misma uno de sus retos fundamentales en el siglo XXI (Soriano Ayala, 2004).

Asimismo, la literatura muestra que las percepciones y actitudes que en el sistema escolar se sostienen sobre algunos grupos sociales (nivel socioeconómico, género, etnia, etc.), tienen un efecto directo sobre las conductas que despliegan al aprender y su logro académico posterior (Navas, Holgado y Sánchez, 2009; Omar et al., 2010).

Al respecto, es importante denotar que en Chile, durante años, la interculturalidad estuvo confinada a lo bilingüe, con programas específicos y referencias en el currículo a propósito de la realidad étnica en su territorio. En este aspecto, existe un avance relevante al reconocerse a nivel de currículo que lo intercultural va más allá de la etnia local, sin embargo, aún no se evidencian sugerencias metodológicas y didácticas que se hagan cargo de lo que se enuncia respecto de la interculturalidad. Se podría decir que existe un rezago respecto de las políticas públicas, asociadas a la inmigración, en los diferentes niveles del Estado (Stefoni, 2011).

Estudios que evalúan la aplicación de la educación intercultural en Chile (UNESCO, 2005) coinciden en señalar las deficiencias que se observan en la implementación de esta política, donde uno de los aspectos identificados como más problemáticos son las bajas expectativas de los docentes respecto de los/as niños/as migrantes. Estos niños son identificados como "grupos en desventaja o vulnerables", para los cuales los docentes plantean estrategias compensatorias de calidad inferior o de exigencias mínimas respecto de los objetivos de aprendizaje comunes. Esto es, las adecuaciones curriculares que se realizan frente al escolar migrante cobran gran relevancia por tanto dicen respecto de la mirada que el docente posee sobre las posibilidades y oportunidades de ese tipo de escolar. Este enfoque estaría guiado por percepciones y estereotipos sociales sobre estos grupos minoritarios (Brown, 2011; Tsaparli-Kitsara y Kaseris, 2006). Así, recabar evidencia sobre la aplicación curricular ante el fenómeno migrante es clave en la búsqueda de acciones que integren y generen espacios inclusivos de acción pedagógica (Enesco et al., 2011; MINEDUC, 2010).

Desde la teoría, el currículum escolar corresponde a la selección cultural, que se transmite a través del sistema escolar (Gvirtz y Palamidessi, 2005). En ese marco, este tiene una doble dimensión, práctica y simbólica. Práctica porque prescribe el contenido de la enseñanza y regula el ejercicio de la docencia, y simbólica, en tanto transmite unas nociones sobre el conocimiento, la cultura y la sociedad (Goodson, 2000). De cierta manera, media entre la conciencia del individuo y la sociedad, y es a través de él que los estudiantes adquieren "una conciencia y una percepciones particulares" sobre las sociedad en la que viven, habitan y se relacionan; y sobre las posibles posiciones que les tocará ocupar dentro de ella (Apple, 1986, p. 50). En este sentido el currículum enmarca una experiencia en que los estudiantes "aprenden y ponen en relación un conjunto de saberes, valores, normas, reglas, concepciones, pensamientos y conocimientos que orientan su actuar" (Quintriqueo, 2010, p. 85).

Desde la perspectiva curricular, las respuestas al desafío de la interculturalidad migrante se manifiestan en esta doble dimensión. Por un parte, en su dimensión práctica, en posibles adaptaciones que permitan que los estudiantes migrantes concilien su experiencia escolar de origen con las prescripciones curriculares que regulan la 
enseñanza y el aprendizaje del sistema educativo de destino; y en su dimensión simbólica, en la transmisión de una concepción dinámica y compleja del concepto de cultura, comprometida con la apreciación de la multiculturalidad como expresión de riqueza social, que aspire a socializar los valores de igualdad, reciprocidad, cooperación e integración (Lluch, 1999), así como a incorporar contenidos relativos a las culturas de origen (Quintriqueo, 2010) y el contexto territorial del cual provienen.

Por lo tanto, es importante recolectar evidencia que demuestre la diversidad de situaciones en la que se encuentran los/as estudiantes migrantes en el sistema escolar chileno. De este modo la investigación en temas de inmigración y educación pueden contribuir a: identificar formas de relación entre el espacio/territorio y su influencia en cómo se desarrollan las medidas inclusivas interculturales; cambiar la percepción que la sociedad tiene frente a los/as migrantes; evidenciar la compleja situación de políticas educativas con las que cuenta el país frente a los temas de migración; así como de las medidas que escuelas con alta población migrante utilizan para "apoyar académicamente a alumnos/as no familiarizados/as con el contexto chileno” (OECD, 2012, p. 179). Contexto que es cultural, pero a su vez socio-territorial.

\section{Entender la indagación socio-territorial}

En la búsqueda de nuevos acercamientos en la comprensión del fenómeno educacionalmigratorio la investigación socio-territorial surge cuando, de manera descriptiva, un espacio se organiza bajo el impulso de sus protagonistas para responder a las necesidades de formación de sus habitantes. Según Lindón (2002) se define una construcción social del territorio realizada por los habitantes locales con las concepciones del mundo, ideas, imágenes que tenían del lugar cuando llegaron, pero que también siguen configurando y organización en la interacción con otros. Este enfoque del territorio, proveniente de la geografía contemporánea, se ha incorporado por su carácter sistémico y multifactorial al enfoque sociológico sobre la socialización, que habla de territorios prescritos (territorios institucionales), territorios vividos (territorios de acción) y territorios soñados (territorios simbólicos); e inclusive territorios interiorizados, relativos al inconsciente colectivo Champollion (2011). Este colectivo está relacionado con la apropiación y vinculación que el sujeto hace del espacio que habita, transitando desde lo funcional de dicho espacio (trabajo, estudio en el mismo) y la carga emotiva asociada a las vivencias en ese espacio (construcción de significados o el sentido que le da el sujeto al espacio). Es decir, el territorio aporta en la conformación de la identidad (Quezada Ortega, 2007), especialmente desde una perspectiva inclusiva que parte por la construcción de conocimiento colectivo, de las vivencias y aportes interculturales en un territorio dado.

Es importante considerar que la toma de conciencia de la interdependencia de los espacios y componentes sociales, permiten captar los pequeños detalles como plantea Selby (1996), lo local está en lo global y lo global en lo local. Se reconoce esta globalidad del mundo, con el objeto de poner en tela de juicio los dogmas existentes sobre dicotomías, distinciones y compartimientos que se suelen utilizar para referirse a los territorios. En este sentido, sobresalen tres ejes fundamentales desde donde estudiar la dimensión socio-territorial: las prácticas sociales, los mecanismos de participación y consumo cultural; y finalmente, ligado a lo anterior, la identidad (Bruner, 2005; Gimenez, 1999; Güell, 2009). 
Esta distinción del territorio y su análisis representa una perspectiva desde el pensamiento sistemático complejo, basándose en el principio de la holografía, que afirma que no sólo las partes están en el todo, sino que el todo también está en las partes, rechazando toda forma de simplificación. Según lo expresa Morin (1994), un territorio no sólo es una constitución de unidades a partir de la biodiversidad, sino una constitución de diversidad interna a partir de las unidades. Esto se comprende en situaciones donde el barrio configura un espacio de interacción de elementos de menor escala (colegio, junta vecinal, plaza, mercados).

El reconocimiento de la dimensión espacial en el marco del modelo de inclusión, permite entender cómo el análisis socio-territorial tributa información y antecedentes necesarios para valorar y poner en relieve fenómenos tan complejos y discutidos como la migración. Lo anterior se entiende como una característica a favor de la comprensión socioterritorial coherente con el nuevo escenario mundial, donde las escalas y las distancias se conjugan aleatoriamente y forman sistemas complejos desde lo social, económico y ambiental. Para Santos (1993), esta dimensión representa la introducción en el sistemamundo de todos los lugares y todos los individuos, aunque en diversos grados.

Cuando hablamos de territorio lo podemos definir como la conjunción entre el espacio y la vida. Tello (2005) plantea que la implicación de ambos conceptos constituye un elemento de análisis social, donde también reconoce que el espacio no es inerte ni estático, en tanto lo constituyen nuestras relaciones saberes, modos de vida, etc. De esta manera el ejercicio investigativo de analizar un territorio determinado, supone enfrentarse a múltiples relaciones que en el pasado no existían o bien se vuelven a resignificar de acuerdo a los nuevos actores y sus respectivas historias de vida que lo conforman. Para Perec (2004, p. 25) "los espacios se han multiplicado, fragmentado y diversificado. Los hay de todos los tamaños y escalas, para todos los usos y todas las funciones. Vivir es pasar de un espacio a otro (...)".

En este sentido el análisis socio territorial es definido por Rossi (1995) como el estudio del conjunto de áreas donde confluyen biografías de vida que necesitan ser indagadas; para los efectos de este escrito, serán consideradas como áreas definidas por su localización geográfica y magnitud sobre el terreno que ocupa, descritas por el tipo de adaptación al crecimiento históricos de las ciudades, comunas o barrios, determinando así su carácter único. Dichas áreas son homogéneas físicas y socialmente, entendiendo la homogeneidad como la estabilidad de los modos de vida en construcciones (migrantes). Una última característica de las áreas está relacionada a la masa y densidad que contienen, o en otras palabras, a la ocupación vertical y horizontal de las construcciones que configuran el territorio. Lo anterior aplica también a los diversos centros educativos y barrios que configuran dichas áreas. En definitiva supone que mientras mudamos formas y estilos de vida, también se generan modificaciones del espacio, no exclusivamente en el modo de uso, sino también en la manera de vivirlo y lo más importante de valorarlo. Por tanto, el análisis del espacio es considerado vital en el contexto territorial, siendo cada vez una fuente de entendimiento de la dominación y sometimiento social y cultural.

La conceptualización de lo socio-territorial ha sido concebida desde la antropología, e inicialmente empleada para comprender las diferentes dinámicas y culturas en una determinada área geográfica. Desde esta perspectiva, el territorio puede ser visto como un contenedor de cultura (Mascareño, 2011). De un modo similar, Castells (1980) se 
refiere a la producción de formas espaciales como el conjunto de procesos que determinan la articulación concreta de elementos materiales sobre un espacio dado, como la organización, las funciones y las actividades que en estos se desarrollen. Dichas definiciones se relacionan en la medida que ven al territorio como anterior a lo social. En este sentido, lo social se crea a partir de las características materiales del territorio, incluyendo la estructura simbólica de los individuos y en sus formas de representar la realidad. Ahora, bien desde la perspectiva de la indagación socio-territorial esta puede abordarse desde una perspectiva donde se acentúan las posibilidades y oportunidades creativas de los actores en constitución, construcción y configuración, son aquellos individuos quienes confieren y reclaman un espacio de análisis y estudio.

Si hablamos de fenómenos socio-territoriales es necesario tener en cuenta dos elementos que son vitales para comprender adecuadamente el concepto y su utilidad. En primer lugar es debido señalar que lo socio territorial puede ser visto como una consecuencia de las distintas dinámicas sociales (como lo es la migración en el sistema escolar) y sus antecedentes. Es decir, el territorio es el resultado y el elemento esencial de la cultura, desde donde se originan y desarrollan determinados tipos de prácticas sociales, clasificables y distinguibles de otros contextos, siendo la migración una de ellas. Por lo anterior hablar de territorio es hablar de cultura socializada, "es todo complejo que incluye el conocimiento, las creencias, el arte, la moral y el derecho, las costumbres y cualquier otro hábito y capacidad adquirida individual y colectivamente por los seres humanos (Taylor, 1958, citado en Kottak, 2002, p. 18). Este proceso de aprendizaje y adaptación cultural depende los símbolos utilizados al interior de una comunidad, son los que crean la cultura y distinguen el territorio y las sociedades que los conforman (Ciccolella, 1999). Por ello que el fenómeno socio-territorial involucra no solo una dimensión geográfica, ni tampoco demográfica, sino hace referencia a un sinnúmero de variables relacionadas a vínculos sociales y a lazos subjetivos que se forman en él. Es, por tanto, un conjunto de relaciones que ocurren en un lugar y espacio (hogar, escuela y barrio) determinado.

La localización y el concepto de espacio social se analizan desde un punto de vista territorial, considerando las prácticas que se dan en su interior siendo ignoradas o tomadas como supuestas. Las prácticas sociales, según De Certeu (2000), pueden tomar dos formas de expresión: las estratégicas y las tácticas. La primera tiene que ver con el conocimiento de un lugar, donde un sujeto decide racionalmente su emplazamiento. En la otra el sujeto carece del concepto de lugar para decidir, ya que "debe actuar con el terreno que le impone y organiza la ley de una fuerza extraña” (De Certeau, 2000, p. 43), por lo que estas se suponen entender como prácticas calculadas, sorpresivas y creativas que se adaptan a las estrategias. Lo anterior releva la importancia de los estudios socio territoriales para comprender la migración, y cómo estos progresivamente, aunque menos intenso que el análisis social, influyen sobre los colectivos espaciales (comunidad escolar y migrantes). Por ejemplo, esto se observa particularmente en el rendimiento académico asociado a la selección de establecimientos educacionales y a las trayectorias de aprendizajes (Coll, 2009), o bien a las experiencias que un determinado contexto genera y transforma en experiencias subjetivas de alto impacto emocional, las cuales, a su vez, plantean una fuerte incidencia en el modo como las personas se comprenden y reconocen a sí mismas.

Por otra parte, la concentración espacial de los migrantes y su respectiva ubicación en centros escolares tiene un carácter multifactorial, donde el precio de la vivienda, los 
mercados laborales locales, o las redes sociales en las que los individuos se insertan (muchas de las que funcionan a partir del principio de la solidaridad étnica) explican por qué nativos y migrantes presentan niveles relativamente altos de segregación espacial en las sociedades de inmigración de nuestro entorno. Esta exclusión se replica, en contextos educativos, donde la escuela juega un rol fundamental en la explicación de fenómenos socio-territoriales y viceversa. En este sentido la inmigración supone ciertos dogmas asociados a las clases sociales los cuales, según Bordieu (1999), radican que las personas no sean capaces de ver que todo esto es posible de cambiar, es decir, la relación entre lugar de residencia-escuela y la posición en el campo social, se transforma en un fenómeno de reificación, donde las personas comienzan a creer que su lugar de residencia-escuela es el que merecen o les corresponde vivir, lo que determina y afecta el imaginario que se puede establecer del espacio, como del otro que convive alrededor y dentro de él. Infante y colaboradores (2013) sugieren que la escuela en la actualidad emerge como un espacio territorializado donde se conjugan áreas de identidad común y dinámicas de apropiación del territorio construidas en prácticas que permiten un uso propio, singular y cotidiano del espacio habitado.

\section{La migración como fenómeno socio-territorial}

Aportes de este enfoque a los procesos de adaptación e inclusión de los migrantes en las escuelas desde una perspectiva intercultural.

Dado lo esbozado en los acápites anteriores, y pensando en la proyección de la indagación intercultural migrante dentro del escenario educativo, se podrían esbozar tres razones del porqué es relevante la sinergia entre lo socio-territorial y la educación inclusiva. En primer lugar, lo socio-territorial se enmarca en una mirada sistémica y multifactorial, que incorpora no solamente lo geográfico, sino las dinámicas que subyacen la habitación de un espacio. En segundo lugar, la perspectiva de territorio que en este artículo se propone considera la subjetividad de los actores, en términos de la apropiación y vinculación de los mismos con el espacio en el que se desarrollan. Dicha perspectiva de vinculación ancla aspectos primordiales de la educación inclusiva tales como la construcción conjunta, la conformación de identidad y la gestación de empatía hacia el otro. Y, en tercer lugar, relaciona a la escuela con su contexto territorial; demarcando elementos de su historia, y de la re-creación que la misma realiza como un espacio de aprendizaje nutrido de las posibilidades que la diversidad cultural provoca.

Por tanto, mirando la relación entre el potencial que se genera desde la unión de la inclusión educativa considerando la territorialidad donde ésta se da, es posible levantar estudios que contribuyan a la política pública a través de la evidencia para constatar lo que sucede actualmente en el caso de la migración y la educación en el país. Este tipo de estudios permitirían entender cómo las escuelas están respondiendo a la nueva realidad migratoria y territorial, para sobre ello construir políticas de apoyo que permitan avanzar hacia prácticas institucionales y pedagógicas inclusivas. Esta constatación es de crucial importancia dada la escasez de políticas que existen al respecto para la generación de programas y políticas educativas en torno al tema. La información generada de indagación con esta mirada, podrían apoyar a oficinas migrantes u otras entidades gubernamentales y no gubernamentales en la comprensión del fenómeno migratorio escolar. 
A nivel de comuna, estudios de corte socio-territorial se convierten en una herramienta de gran valor para conocer, monitorear y hacer seguimiento a aquellos establecimientos educacionales con mayor índice de migración escolar; así como establecer una mirada panorámica respecto de las condiciones de caracterización social que existen en los establecimientos educacionales de dichas comunas. Esto permitiría la generación de comunidades educativas que reconozcan el territorio como un factor que configura las trayectorias académicas (biográfica) de sus estudiantes.

A su vez, los resultados socio territoriales podrían ir acompañados de análisis de implementación curricular, lo cual permitirá reconocer mecanismos de prevención y fortalecimiento de programas de inclusión pedagógica, mostrando tensiones, necesidades en torno a la adecuación del escolar migrante; así como permitirá contar con ejemplos de procesos exitosos de integración y aplicación de currículo intercultural, basados en la reconstrucción de las características locales, transformadas por el influjo migratorio.

El tipo de información tiene el potencial de orientar a las escuelas en el diseño de estrategias para generar espacios inclusivos con actividades concretas de promoción de la diversidad cultural y de estrategias pedagógicas asociadas. El concepto de espacio heterogéneo nos recuerda y enfrenta a una realidad con diversos posibles contextos, y desafíos. Uno de ellos es mirar el proceso de Formación Inicial Docente (FID) y su transferencia al ámbito escolar posterior, como espacio de inclusión. La mirada de lo socio-territorial aporta a la discusión de la FID en la medida que amplía el foco de lo identitario y cultural no solamente a aspectos propios de las creencias y expectativas de un sujeto o colectivo, sino también de cómo el territorio enmarca dichas creencias y facilita experiencia que permitan reconfigurar dichas identidades. Esto es de suma relevancia, ya que implica dinamizar todos los elementos del proceso de socialización de una persona hacia sí mismo y los demás, condicionado por el espacio que habitan y al cual pertenecen. Esto, a su vez, determina su modo de ver el mundo y situarse en él, su sistema de creencias y sus relaciones, su concepción de las normas, derechos y deberes hacia los demás.

Finalmente, los resultados apoyarían la visibilización del fenómeno de la migración escolar, fortaleciendo la toma de decisiones de índole político, basado en evidencia, y bajo la consideración de migración como un fenómeno multifactorial. Lo anterior permitiría crear un espacio de diálogo, desde la información aportada por el análisis socio territorial, y su aplicación a los procesos educativos de los niños, niñas y adolescentes migrantes; potenciando su impacto.

El objetivo de la inclusión intercultural del migrante al sistema educacional, ampliamente compartida, enfrenta serios desafíos que deben ser abordados con la incorporación de nuevas propuestas y miradas desde la formación inicial y continua de los profesores, incluyendo la forma en que las expectativas, representaciones e identidades se organizan y conforman territorialmente, para luego ser vivenciadas al interior de la escuela. Este artículo promueve el uso de la mirada de lo socio-territorial al diseño de políticas públicas en materia de inclusión del escolar migrante; así como en el diseño de orientaciones que nutran las estrategias que actualmente utilizan las escuelas. La educación aspira a promover equidad e inclusión en las oportunidades de aprendizaje en un clima de aceptación y respeto entre quienes conforman las comunidades educativas. La conformación territorial releva la construcción identitaria de dichas relaciones educacionales. 


\section{Referencias}

Aguado. J. y Ariosa, M. (1991). Ideología, identidad y cultura: tres elementos básicos en la comprensión de la reproducción cultural. Boletín de Antropología Americana, 23, 67-82.

Ainscow, M., Booth, T. y Dyson, A. (2006). Improving schools developing inclusion. Londres: Routledge.

Alegre, M. A., Benito, R. y González, S. (2006). Immigrants als instituts: L'acollida vista pels seus protagonistes. Barcelona: Fundación Jaume Boffil.

Apple, M.W. (1986). Ideología y currículo. Madrid: Akal.

Arias, G., Moreno, R. y Núñez, D. (2010). Inmigración latinoamericana en Chile: Analizando perfiles y patrones de localización de la comunidad peruana en el área metropolitana de Santiago. Revista Tiempo y Espacio, 25, 1-16.

Aronson, J., Quinn, D. M. y Spencer, S. J. (1998). Stereotype threat and the academic underperformance of minorities and women. En C. Stangor y J. K. Swim (Eds.), Prejudice: The target's perspective (pp. 83-103). San Diego, CA: Academic Press

Avramidis, E. y Norwich, B. (2002) Teachers' attitudes towards integration/inclusion: A review of the literature. European Journal of Special Needs Education, 17(2), 129-14.

Baron, R. A. y Byrne, D. (2005). Prejuicio: Causas, efectos y formas de contrarrestarlo. En R. A. Barón y D. Byrne (Eds.), Psicología social (pp. 215-261). Madrid: Prentice Hall

Berrios-Valenzuela, L. A. y Palou-Julián, B. (2015). Educación intercultural en Chile: La integración del alumnado extranjero en el sistema escolar. Educación y Educadores, $17(3), 1$ 24 .

Berry, J. W. y Vedder, P. (2016). Adaptation of immigrant children, adolescents, and their families. Childhood and Adolescence: Cross-Cultural Perspectives and Applications, 321, 34-54,

Blanco, R. (2006). La equidad y la inclusión social: Uno de los desafíos de la educación y la escuela hoy. REICE. Revista Iberoamericana sobre Calidad, Eficacia y Cambio en Educación, 4(3), 1-15.

Bodenhausen, G., Kramer, G. y Susser, K. (1994). Happiness and stereotypic thinking in social judgment. Journal of Personality and Social Psychology, 66, 62 1-632.

Booth, T. y Ainscow, M. (2012). Guía para la inclusión educativa. Desarrollando el aprendizaje y la participación en las escuelas. Santiago: Fundación Creando Futuro.

Bourdieu, P. (1999). La miseria del mundo. Madrid: Akal.

Brown, C. S. (2011). American elementary school children's attitudes about immigrants, immigration, and being an American. Journal of Applied Developmental Psychology, 32, 109117.

Bruner, E. M. (2005). Culture on tour: Ethnographies of travel. Chicago, IL: University of Chicago Press.

Callejas, C., Solbes, I., Dopico, C. y Escudero, A. (2011). Estudio de las actitudes inter-étnicas en la infancia a través de una medida implícita. Anales de Psicología, 27, 655-661.

CASEN. (2006). Encuesta de caracterización socioeconómica nacional. Santiago: Ministerio de Planificación.

CASEN. (2013). Encuesta de caracterización socioeconómica nacional. Santiago: Ministerio de Desarrollo Social. 
Castells, M. (1980). Cidade, democracia e socialismo: a experiência das associações de vizinhos de Madri. Rio de Janeiro: Paz e terra.

Castells, M. (2001). La era de la información. Madrid: Alianza.

Cárdenas, M., Gómez, F., Méndez, L. y Yañez, S. (2011). Reporte de los niveles de prejucio sutil y manifiesto hacia los inmigrantes bolivianos y análisis de su relación con variables psicosociales. Psicoperspectivas. Individuo y Sociedad 1O(1), 125-143.

Champollion, P. (2011). El impacto del territorio en la educación. El caso de la escuela rural en Francia. Profesorado. Revista de Curriculum y Formación del Profesorado, 15(2), 53-69.

Ciccolella, P. (1999). Globalización y dualización en la región metropolitana de Buenos Aires: Grandes inversiones y reestructuración socioterritorial en los años noventa. EURE, 25(76), 5-27.

Coll, C. (2009). Enseñar y aprender en el siglo XXI: El sentido de los aprendizajes escolares. Calidad, equidad y reformas en la enseñanza. Madrid: OEI.

Degner, J. y Wentura, D. (2010). Automatic prejudice in childhood and early adolescence. Journal of Personality and Social Psychology, 98, 356-374.

del Río, F. y Balladares, J. (2010). Género y nivel socioeconómico de los niños: Expectativas del docente en formación. Revista Psykhe, 19, 81-90.

Donoso, A., Mardones, P. y Contreras, R. (2009). Propuestas y desafíos a partir de la experiencia de una escuela con migrantes en el Barrio Yungay, Santiago de Chile. Docencia, 37, 56-62.

Dovidio, J. F. (2001). On the nature of contemporary prejudice: The third wave. Journal of Social Issues, 57, 829-849.

Echeita, G., Simón, C., Verdugo, M., Sandoval, M., López, M. y Calvo, I. (2009). Paradojas y dilemas en el proceso de inclusión educativa en España. Revista de Educación, 349, 153-178.

Enesco, I., Guerrero, S., Lago, M. y Rodríguez, P. (2011). How stable is children's affective orientation toward different ethnic groups? A longitudinal study of in-group and outgroup attitudes among preschoolers. Anales de Psicología, 27, 631-638.

Giménez, G. (1999). La importancia estratégica de los estudios culturales en el campo de las ciencias sociales. R. Reguillo y R. Fuentes Navarro (Coords.), Pensar las ciencias sociales hoy (pp. 71-98). Guadalajara: ITESO.

Giménez, G. (2001). Cultura, territorio y migraciones. Aproximaciones teóricas. Alteridades, $11(22), 5-14$.

Goffman, E. (2001). Estigma. La identidad deteriorada. Buenos Aires: Amorrortu Editores.

González, R. (2009). Antecedentes psicológicos de las dinámicas intergrupales entre chilenos e inmigrantes peruanos. Recuperado desde http://www.cpce.cl/

Goodson, I. (2000). El cambio en el curriculum. Buenos Aires: Editorial Octaedro.

Güell, P. (2009). El arte de clasificar a los chilenos. Santiago: Ediciones UDP.

Guerrero, E., Provoste, P. y Valdés, A. (2006). La desigualdad olvidada: Género y educación en Chile. Recuperado de http://www.lppuerj.net/olped/documentos/1830.pdf

Gvirtz, S. y Palamidessi, M. (2005). El ABC de la tarea docente: Currículum y enseñanza. Buenos Aires: Aique Grupo Editor.

Hitti, A., Lynn Mulvey, K. y Killen, M. (2011). Social exclusion and culture: The role of group norms, group identity and fairness. Anales de Psicología, 27, 587-599. 
INE. (2003). Síntesis de resultados. Censo 2002. Santiago: Instituto Nacional de Estadísticas.

INE. (2015). Compendio estadístico 2015. Santiago: Instituto Nacional de Estadísticas.

Infante, M., Matus, C., Paulsen, A., Salazar, A. y Vizcarra, R. (2013). Narrando la vulnerabilidad escolar: Perfomatividad, espacio y territorio. Literatura y Lingüística, 27, 281-308.

Kottak, C. P. (2002). Antropología cultural. Londres: McGraw-Hill.

Lindón, A. (2002). Trabajo, espacios de vida y cotidianidad. La periferia oriental de la ciudad de México. Scripta Nova Revista Electrónica de Geografía y Ciencias Sociales, 6(119), 1-23.

León, B., Mira, A. y Gómez, T. (2007). Evaluación de las opiniones sobre la inmigración y la multiculturalidad en la escuela de alumnos de magisterio. Revista Electrónica de Investigación Psicoeducativa 5, 259-282.

Lowery, B. S., Hardin, C. D. y Sinclair, S. (2001). Social influence effects on automatic racial prejudice. Journal of Personality and Social Psychology, 81, 842-856.

Lluch, X. (1999). Plural. Educació intercultural. Valencia: Tàndem edicions.

Mähönen, T. A. y Jasinskaja-Lahti, I. (2016). Meet me halfway: Socio-cultural adaptation and perceived contact willingness of host nationals as predictors of immigrant's threat perceptions. Journal of Community \&' Applied Social Psychology, 26(2), 125-135.

Mardones, P. (2010). Volveré y seré millones. Migración y etnogénesis. Buenos Aires: Universidad de Buenos Aires.

Mascareño, A. (2011). Sociología de la intervención: Orientación sistémica contextual. Revista Mad, 25, 1-33.

McGarty, C., Lala, G. y Douglas, K. M. (2011). Opinion-based groups. En Z. Birchmeier (Ed.), Strategic uses of social technology: An interactive perspective of social psychology, 145171. Londres: Cambridge University Press. doi: 10.1017/CBO9781139042802.008

Ministerio del Interior. (2004). Estadísticas migratorias. Recuperado desde http://www.extranjeria.gob.cl/filesapp/censo__2002_.pdf

MINEDUC. (2005). Oficio $N^{\circ} 7 / 1008$ (1531). Recuperado desde http://www.extranjeria.gob.cl/filesapp/Of_07_1008_MINEDUC.pdf

MINEDUC. (2010). Educar en la diversidad. Chile: UNESCO.

Moreno L. y Oyarzun, F. (2013). Interculturalidad en el sistema educacional chileno? Representaciones sociales de la integración de estudiantes extranjeros en dos establecimientos educacionales municipales. Revista Electrónica de Psicología Iztacala, 16(3), 764-790.

Morin, E. (1994). La complejidad y la acción. En E. Morin (Ed.), Introducción al pensamiento complejo (pp. 14-31). Barcelona: Gedisa.

Murillo, J. (2010). Liderazgo para la inclusión escolar y la justicia social. Aportaciones de la Investigación. Revista Latinoamericana de Educación Inclusiva, 4(1), 7-33.

Navas, L., Holgado, F. y Sánchez A. (2009). Predicción de los estereotipos académicos ante los estudiantes inmigrantes. Horizontes Educacionales, 14, 37-47.

OBIMID. (2016) La migración en Chile: breve reporte y caracterización. Santiago: OBIMID.

OECD. (2012). International migration Outlook 2012. Paris: OECD.

OIM. (2006). Derecho internacional sobre migración $n^{\circ}$ 7. Glosario sobre migración. Recuperado de http://publications.iom.int/ 
OIM. (2007). Los migrantes, sus derechos y la legislación aplicable. Guía práctica. Recuperado de http://www.iom.int/

Olmos A. (2012). Segregaciones y construcción de la diferencia en la escuela. Madrid: Trotta

Omar, A., Urteaga, A., Uribe, H. y Nilton, N. (2010). Capital sociocultural familiar, autoestima y desempeño académico en adolescentes. Revista Latinoamericana de Estudios Educativos 60, 93-114.

Ortiz Cobo, M. (2008). Inmigración en las aulas: Percepciones prejuiciosas de los docentes. Papers: Revista de Sociología, 87, 253-268.

Pavez, I. (2012). Inmigración y racismo: Experiencias de niñez peruana en Santiago de Chile. Si Somos Americanos 12(1), 75-99

Perec, G. P., Perec, M. G., Corea, C., Cantarelli, I., Halperín Donghi, T., Donghi, T. H., ... y Rodríguez-Morán, M. (2012). Tentativa de agotamiento de un lugar parisino (No. 840-3). París: Christian Bourgois

Pettigrew, T. F., y Meertens, R. W. (1995). Subtle and blatant prejudice in western Europe. European Journal of Social Psychology, 25(1), 57-75.

Poblete Acuña, C. y Zegers Quiroga, N. (2014). Migración y extranjería. Análisis crítico del Proyecto de Ley de junio del año 2013. Recuperado de http://www.repositorio.uchile.cl/handle/2250/129757

Portes, A. (1997). Economic sociology and the sociology of immigration: A conceptual overview. En A. Portes (Ed.), The economic sociology of immigration (pp.1-41). Nueva York, NJ: Russell Sage Foundation.

Quezada Ortega, M. (2007). Migración, arraigo y apropiación del espacio en la recomposición de identidades socioterritoriales. Cultura y Representaciones Sociales, 2(3), 23-39.

Quintriqueo, S. (2010). Implicancias de un modelo curricular monocultural en contexto mapuche. Santiago: Gráfica LOM.

Rodríguez, H. y Retortillo, A. (2006). El prejuicio en la escuela. Un estudio sobre el componente conductual del prejuicio étnico de alumnos de quinto de primaria. Revista Interuniversitaria de Formación del Profesorado, 20, 133-149.

Rossi, G. (1995). The object-oriented hypermedia design model. Communications of the ACM, $38(8), 45-46$.

Salas, N., del Río, F., Kong, F y San Martin, C. (2016). Caracterización y prejuicio acerca de los inmigrantes en el sistema escolar. Santiago: Ministerio de Educación.

Santos, M. A. (1993). Los espacios de la globalización. Anales de geografía de la Universidad Complutense, 13, 69-77.

Schwartz, S. J., Kim, S. Y., Whitbourne, S. K., Zamboanga, B. L., Weisskirch, R. S., Forthun, L. F. y Luyckx, K. (2013). Converging identities: Dimensions of acculturation and personal identity status among immigrant college students. Cultural Diversity and Ethnic Minority Psychology, 19, 155-171.

Segura, M. y Abde, K. (2014). Barrios y población inmigrantes: el caso de la comuna de Santiago. Revista INVI, 29, 19-77.

Selby, D. (1996). Educación global: Hacia una irreducible perspectiva global en la escuela. Revista Educación, 16(2), 5-12.

Shmelkes, S. (2004). La educación intercultural: un campo en proceso de consolidación. Revista Mexicana de Investigación Educativa, 9, 9-13 
Sirlopú, D., Melipillán, R., Sánchez, A. y Valdés, C. (2015). ¿Malos para aceptar la diversidad? Predictores socio-demográficos y psicológicos de las actitudes hacia el multiculturalismo en Chile. Psykhe, 24(2), 1-13.

Soriano Ayala, E. (Coord.). (2004). La práctica educativa intercultural. Madrid: La Muralla.

Stangor, C. (2009). The study of stereotyping, prejudice, and discrimination within social psychology: A quick history of theory and research. En T. D. Nelson (Ed.), Handbook of prejudice, stereotyping, and discrimination (pp. 1-22). Mahwah: Lawrence Erlbaum Associates.

Stefoni, C. (2011). Perfil Migratorio de Chile. Santiago: Organización Internacional de las Migraciones.

Stefoni, C., Acosta, E., Gaymer, M. y Casas-Cordero, F. (2008). Niños y niñas inmigrantes en Santiago de Chile. Entre la integración y la exclusión. Santiago: Universidad Alberto Hurtado.

Stefoni, C., Acosta, E., Gaymer, M. y Casas-Cordero, F. (2010). El derecho a la educación de los niños y niñas inmigrantes en Chile. Universidad de Deusto; Deustuko Unibertsitatea.

Steinmayr, R. y Spinath, B. (2008). Sex differences in school achievement: What are the roles of personality and achievement motivation? European Journal of Personality, 22, 185-209.

Tello, E. (2005). La historia cuenta: del crecimiento económico al desarrollo humano sostenible. Santiago: Editorial El Viejo Topo.

Thayer, L. E. (2008). La expropiación del tiempo y la apropiación del espacio. La incorporación de inmigrantes latinoamericanos a la Comunidad de Madrid (Tesis doctoral). Universidad Complutense de Madrid.

Theoharis, G. T (2007). Social justice educational leaders and resistance: Toward a theory of social justice educational leadership. Educational Administration Quartely, 43, 22 1-258.

Tijoux, M. E. (2013). Las escuelas de la inmigración en la ciudad de Santiago: Elementos para una educación contra el racismo. Polis 12(35), 287-307.

Tijoux, M. E. (2014). La extranjeridad inmigrante peruana en Chile. Revista Observatorio Cultura, 4, 9-12.

Tropp, L. R. (2003). The psychological impact of prejudice: Implications for intergroup contact. Group Processes $\mathbb{E}^{\circ}$ Intergroup Relations, 6, 131-149.

Tsaparli-Kitsara, A. y Kaseris, M. (2006). Autoestima e inmigración. Revista Interuniversitaria de Formación del Profesorado 20, 191-206.

UNESCO. (1994). The Salamanca statement and framework for action on special needs education. Paris: UNESCO.

UNESCO. (2005). Políticas educativas de atención a la diversidad. Santiago UNESCO.

Veredas, S. (1999). Procesos de construcción de identidad entre la población inmigrante. Papers: Revista de Sociología 57, 113-129.

Walsh, C. (2005). Interculturalidad, conocimientos y decolonialidad. Signo y Pensamiento, 24(46), 39-50.

Zafra, R. (2011). La diversidad en las aulas como factor de cambio social. Cuadernos de Educación y Desarrollo, 3(25), 1-24. 


\section{Breve CV de los autores}

\section{Natalia Salas Guzmán}

Ph.D. en Psicología por la Pontificia Universidad Católica de Chile y es Psicóloga por la Universidad de Costa Rica. Es profesora e investigadora de temáticas asociadas a la inclusión, migrantes, cognición social, neurociencias y diversidad. Dichas líneas de investigación ha sido financiadas por fondos nacionales e internacionales (MINEDUC, CONICYT, OEA y BID). Actualmente es académica e investigadora de la Facultad de Educación de la Universidad Diego Portales (Chile) y coordina el Observatorio Chileno de Inclusión Educativa Migrante (OCIEM). ORCID ID: 0000-0001-8815-5979. Email: natalia.salas@mail.udp.cl

\section{Felipe Kong López}

Doctor en Didáctica de las Ciencias y Educación Ambiental (Universidad Autónoma Barcelona), Geógrafo y licenciado en Geografía (Pontificia Universidad Católica). Coordina el área de Didáctica de las Ciencias (UDP). Su línea de investigación se centra en las concepciones de estudiantes y profesores acerca de la Enseñanza de las Ciencias y socio-territorialidad. Actualmente es académico e investigador de la Facultad de Educación de la Universidad Diego Portales, en Santiago de Chile y parte del equipo del Observatorio Chileno de Inclusión Educativa Migrante (OCIEM). ORCID ID: Ooooo002-8914-3336. Email: felipe.kong@udp.cl

\section{Renato Gazmuri Stein}

Doctor en Didáctica de la Historia, la Geografía y las Ciencias Sociales (Universidad Autónoma de Barcelona) y Licenciado en Historia (Pontifica Universidad Católica de Chile). Sus principales líneas de investigación son la teoría curricular, la enseñanza y el aprendizaje escolar de la historia, la sociedad y la ciudadanía, y formación docente en Didáctica de la Historia y las Ciencias Sociales. Actualmente es académico e investigador de la Facultad de Educación de la Universidad Diego Portales y parte del equipo del Observatorio Chileno de Inclusión Educativa Migrante (OCIEM). ORCID ID: 00000002-0927-875X. Email: renato.gazmuri@udp.cl 